\title{
On the Structure of Electrons and Other Charged Leptons
}

\author{
M. E. Shulman \\ Dnepr (Dnepropetrovsk), 49054, Ukrainskaya, Ukraine \\ Email: shulmanme37@gmail.com
}

How to cite this paper: Shulman, M.E. (2017) On the Structure of Electrons and Other Charged Leptons. Journal of High Energy Physics, Gravitation and Cosmology, 3, 503-521.

https://doi.org/10.4236/jhepgc.2017.33039

Received: April 6, 2017

Accepted: July 28, 2017

Published: July 31, 2017

Copyright (C 2017 by author and Scientific Research Publishing Inc. This work is licensed under the Creative Commons Attribution International License (CC BY 4.0).

http://creativecommons.org/licenses/by/4.0/ (c) (i) Open Access

\begin{abstract}
A model of the electron is examined, allowing us to obtain its mass, spin and magnetic moment. The electron is represented as a sphere of classical radius (protoelektron) with zero rest mass, the rotating orbit radius of which is reduced value of the Compton wavelength of the electron. The ratio of the radius of the sphere to the radius of the orbit is equal to the fine structure constant. The sphere has a single charge distributed over its surface. Due mutual repulsion of parts of charge sphere acquires a mass equal to half of the rest mass of an electron, rotating mechanical mass protoelektron on orbit provides its characteristic electron spin $1 / 2$ and kinetic energy, which creates $1 / 4$ of the rest mass. Rotation of charge, similar to the ring current generates a magnetic moment equal to the Bohr magneton and magnetic energy, creating $1 / 4$ of the rest mass of an electron. The total energy of the electron is the sum of its electrostatic, magnetic and kinetic energy. Accordingly, the total mass of the electron is the sum of the masses of electrostatic, magnetic and kinetic origin. The model is applicable to the muon and tau leptons. The correct ratio between the mass, spin and magnetic moment for them observed under the condition in the ratio of the radius of the charged sphere to the radius of the orbit equal to the fine structure constant. The model allows us to understand the physical nature of a number of problems: the Heisenberg uncertainty principle, Lorentz transformations and wave properties of the electron. The cause of the orbital rotation proto-particles is a magnetic field which creates self-acting rotation proto-particles around its own axis.
\end{abstract}

\section{Keywords}

Electron Structure, Mass, Spin, Magnetic Moment

\section{Introduction}

Electron according to modern scientific ideas is an elementary, t. e. without in- 
ner structure, particle. Its dimensions are supposed to be very small, less than $10^{-17} \mathrm{~m}$, the so-called material point. Such views entail a lot of problems. Insignificant size should correspond to the great mass, whereas the mass of the electron is measured with great accuracy and meets its classical radius. It is necessary to create a theory of mass renormalization. It is more difficult to explain its own torque-the spin of a material point and closely associated magnetic moment, and therefore it had to declare them "purely quantum properties", inaccessible to human consciousness. All of these issues remain unresolved for over a century. Many studies have attempted to create a model of the electron, corresponding to one or more of these parameters. An overview of such research works is given in [1] [2] [3].

Assertion that it is impossible to imagine the properties of the electron unfairly limits the ability of the human mind. The presence of the final mass of the electron contradicts the idea of an electron as a material point. The presence of the spin and magnetic moment are evidence about rotating some charged masses. Uncertainty relation coordinate and momentum, the dimension and composition of the Planck constant, simple relations between this value and the properties of the electron probably led many researchers to attempt to build on this basis, different models of the structure of this particle. Any physicist, even using the most complicated of mathematics, consciously or subconsciously imagines model of the object or process, even if modelt was not fully satisfied with his. Many of the models are not able to explain all the properties of the electron, either because of the attachment to the compact structure, or because of the limited existing stereotypes, for example, point-electron size, inability to speed of light of a massive movement of the body which is impossible without radiation rotation, and so on. However, outlines in this article calculation show that it is the rejection of these restrictions that will result in success. This leads to the search for and the justification of the ways to circumvent these restrictions.

We used the values of the fundamental physical constants of the tables [4]:

$h=6.6206957 \cdot 10^{-34} \mathrm{~J} \cdot \mathrm{s}$-Planck's constant;

$\hbar=\frac{h}{2 \pi}=1.054571726 \times 10^{-34} \mathrm{~J} \cdot \mathrm{s}-$ Planck constant over $2 \mathrm{pi}$;

$c=2.99792458 \times 10^{8} \mathrm{~m} \cdot \mathrm{s}^{-1}$-The speed of light in vacuum;

$\alpha=7.2973525698 \times 10^{-3}$-Fine structure constant;

$\varepsilon_{0}=8.854187817 \times 10^{-12} \mathrm{~F} \cdot \mathrm{m}^{-1}$-Electric constant;

$\mu_{0}=4 \pi \times 10^{-7}=12.566370614 \times 10^{-7} \mathrm{~N} \cdot \mathrm{A}^{-2}$-Magnetic constant;

$e=1.602176565 \times 10^{-19} \mathrm{C}$-Elementary charge;

$r_{e}=2.8179403267 \times 10^{-15} \mathrm{~m}$-Classical electron radius;

$m_{e}=9.10938291 \times 10^{-31} \mathrm{~kg}$-Mass of the electron;

$E_{e}=m_{e} c^{2}=8.18710506 \times 10^{-14} \mathrm{~J}=0.510998928 \mathrm{MeV}$-The energy equivalent of the mass of the electron;

$\lambda_{c e}=2.4263102389 \times 10^{-12} \mathrm{~m}$-Compton wavelength of the electron;

$\lambda_{c e}=\frac{\lambda_{\text {ce }}}{2 \pi}=386.15926800 \times 10^{-15} \mathrm{~m}$-Compton wavelength of the electron over 
$2 \mathrm{pi}$

$\mu_{b}=\frac{h e}{4 \pi m_{e}}=927.400968 \times 10^{-26} \mathrm{~J} \cdot \mathrm{T}^{-1}$-Bohr magneton.

All calculations were performed in the SI system.

Note that experimentally measured the electron Compton wavelength corresponds to the known formula [5]

$$
\lambda_{c e}=\frac{h}{m_{e} c}
$$

Which implies that the mysterious Planck constant can be represented by the product of three fundamental constants:

$$
h=m_{e} \lambda_{c e} c
$$

It is also conceivable that $\lambda_{c e}$ there circumference whose radius is equal to

$$
\lambda_{c e}=\frac{\lambda_{c e}}{2 \pi}
$$

In what follows we call this quantity the Compton radius of the electron:

$$
R_{c e}=\lambda_{c e}
$$

Expanded form of Planck's constant takes the following form:

$$
h=m_{e} 2 \pi R_{c e} c
$$

Constant Dirac has:

$$
\hbar=m_{e} R_{c e} C
$$

Attitude classic radius of an electron to its Compton radius equal to the fine structure constant*:

$$
\frac{r_{e}}{R_{c e}}=7.2973525698 \times 10^{-3}=\frac{1}{137.0359990743}=\alpha
$$

(We note in passing that the ratio of the Compton electron radius to the radius of the first Bohr orbit is also equal to the fine structure constant, otherwise known as the Sommerfeld constant). Note that the spin of an electron requires that some of the factors in the Planck constant were equal to half of the tabulated value

$$
s=\frac{\hbar}{2}=\frac{m_{e} R_{c e} c}{2}
$$

Consequently, we can assume that a particle with a mass equal to half the mass of the electron spins at the speed of light in orbit of Compton radius. Study this model (see. Section 3) confirmed that so you can get not only spin, but also the magnetic moment of the electron, and further the remaining half of the mass. Confirmation of the assumption of a particle is G. V. Nikolaev [9]: "In the modern electrodynamics has not yet been resolved contradiction with the nature of the rest mass $m_{0}$ and the electron charge e. If the total energy of the electron $W_{0}$

${ }^{*}$ I was able to establish this relationship in the early 70 -ies. For the first time it was published in June 2009 in the article [6]. However, I met this ratio the October 26, 2012 in the book of A. G. Kyryako [7]. This relation is given in [8]. 
corresponds to the rest mass of the electron $m_{0}=\frac{w_{0}}{c^{2}}$, the energy of the electric field $W_{E}$ corresponds to the mass of the electron $m_{E}=\frac{1}{2} m_{0}$ ". The same encounter in L. D. Landau and E. M. Lifshits [10]: “... the expression for the energy of the system of charges":

$$
U=\frac{1}{2} \int \rho \phi \mathrm{d} V
$$

And finally, fully convinced detailed explanation R. Feynman et al. [11] that deserves to bring it in full: Imagine that we have a very simple model of the electron, when all his charge $q$ is uniformly distributed over the surface of a sphere of radius a. [...] Now we calculate the energy electromagnetic field. If the charge is stationary, then there is no magnetic field around there, and the energy per unit volume is proportional to the square of the electric field. The magnitude of the electric field is equal $q / 4 \pi \varepsilon_{0} r^{2}$, so that the density per unit volume

$$
u=\frac{\varepsilon_{0}}{2} E^{2}=\frac{q^{2}}{32 \pi^{2} \varepsilon_{0} r^{4}}
$$

To obtain the total energy density of this need to integrate over the whole space. Using the volume element $4 \pi r^{2} \mathrm{~d} r$, we find the total energy, which we denote by Uel:

$$
\text { Uэл }=\int \frac{q^{2}}{8 \pi \varepsilon_{0} r^{2}} \mathrm{~d} r
$$

This expression is very easy to integrate. Lower limit of integration is a, and the upper-infinity, so

$$
\text { Uэл }=\frac{1}{2} \frac{q^{2}}{4 \pi \varepsilon_{0}} \frac{1}{a} .
$$

And further: The value of

$$
r_{0}=\frac{e^{2}}{m c^{2}}
$$

It called "classical electron radius" and is equal to $2.82 \times 10^{-13} \mathrm{~cm}$, see, that is one hundred-thousandth the diameter of an atom. [...] Instead of arguing which distribution is right and what is not, it was decided to take as the "nominap" value of radius $r_{0}$. A different theory ascribe its coefficient. The electrostatic energy of a particle is inversely proportional to its radius. If the electron is represented as a material point energy value becomes infinitely large, and requires an artificial of mass renormalization. Thus, it would seem, there is enough reason to believe that the scope of the classical radius of the electron charge has a mass equal to half the mass of the electron and having an electrostatic origin.

However, in the above-cited statement Feynman talking about the energy field associated with the charge of an electron, but this value is related to the energy of the electron, it remains somewhat unclear. If we assume that the material basis is the scope of the classical electron radius and distributed on the surface of 
the elementary charge, the mass of the sphere is natural to associate with potential energy auto repulsion charge. More Henri Poincare concerned by the question, why the charge does not fly apart under the forces of repulsion. Therefore, the mysterious forces that ensure stability of the electron, known in physics as the "stresses of Poincaré".

To calculate the energy of Coulomb repulsion of the charge necessary to find the sum of the interactions of each section of the charged sphere with all the others. We assume that the field is filled matter of the physical vacuum with electric constant $\varepsilon_{0}$. On a spherical surface we choose an infinitesimal portion of the point A (Figure 1) with the charge $\Delta e$.

This charge interacts with a charge ring infinitesimal width $\mathrm{d} l=r_{0} \mathrm{~d} \varphi$. The radius of the ring $r_{1}=r_{0} \sin \varphi$, the charge density $\rho=\frac{e}{4 \pi \cdot r_{0}^{2}}$. Consequently, the charge rings

$$
\mathrm{d} e=\rho \mathrm{d} S=\frac{e}{4 \pi \cdot r_{0}^{2}} \cdot 2 \pi \cdot r_{0} \sin \varphi \cdot r_{0} \mathrm{~d} \varphi=\frac{e}{2} \sin \varphi \mathrm{d} \varphi
$$

The distance between the charge at point $A$ and any point of the ring-chord

$$
A B=2 r_{0} \sin \frac{\pi-\varphi}{2} .
$$

The interaction energy between the charge and the charge of the ring at point $A$

$$
\mathrm{d} E=\frac{\Delta e \cdot e}{4 \pi \varepsilon_{0} \cdot 2 r_{0} \sin \left(\frac{\pi-\varphi}{2}\right) \cdot 2} \sin \varphi \mathrm{d} \varphi .
$$

where the energy of interaction between the charge at point $A$ and all the other charges is

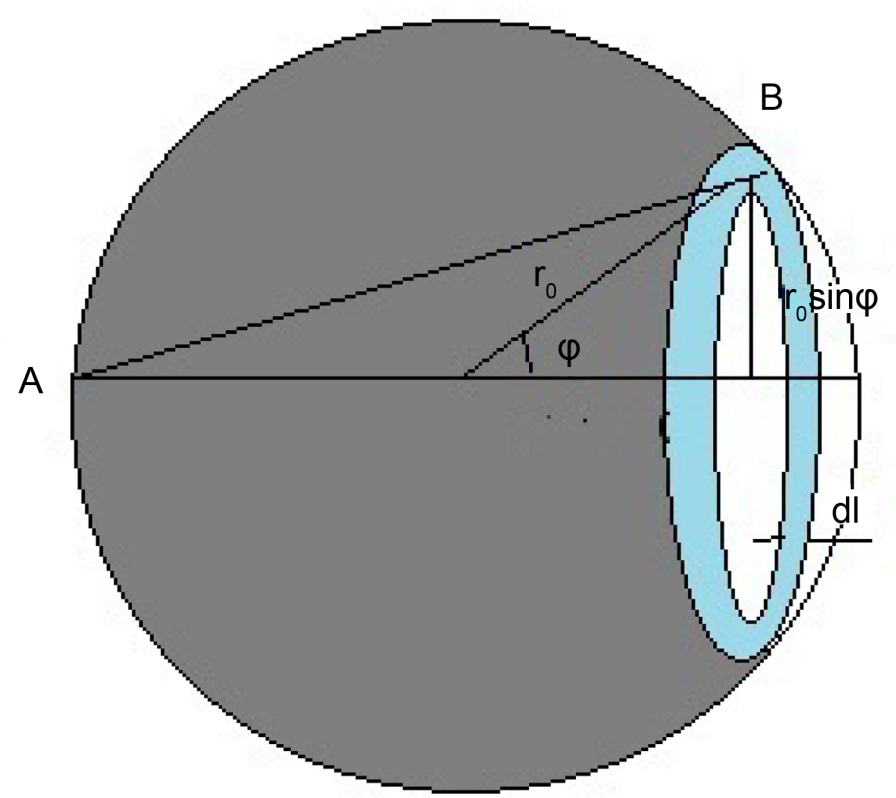

Figure 1. Scheme for calculating the self-repulsion energy of an electron. 


$$
E_{A}=\frac{\Delta e \cdot e}{16 \pi \varepsilon_{0} r_{0}} \int_{0}^{\pi} \frac{\sin \varphi}{\sin \left(\frac{\pi-\varphi}{2}\right)} \mathrm{d} \varphi=\frac{\Delta e \cdot e}{16 \pi \varepsilon_{0} r_{0}} \cdot 4=\frac{\Delta e \cdot e}{4 \pi \varepsilon_{0} r_{0}}
$$

In order to avoid double counting of interactions in the calculation of the total energy of repulsion necessary amount of private charges set equal to half of the elementary charge protoelektrona: $\sum \Delta e=\frac{e}{2}$. Then the total energy of repulsion

$$
E=\frac{1}{2} \cdot \frac{e^{2}}{4 \pi \varepsilon_{0} r_{0}}
$$

As you can see, the internal potential energy of Coulomb repulsion of the charge is exactly equal to the energy of its external electric field. This has two important implications:

1) Equivalent to the mass of the charged spheres its internal energy, whereas the equivalent field energy is apparently weight field;

2) The energy field, which is calculated as the work of the delivery charge from infinity to the surface of a sphere, can be interpreted as the energy of the charge retention of scattering into infinity. Thus, the voltage Poincare this pressure on the electron from its own external electric field. One of the main objections to the proposed model, the electron is the inability of a particle having a mass move at the speed of light. But the motion of a massive particle with the speed of light is permissible if its rest mass is zero, is an example of a photon. Such particles getting energy from outside, simultaneously obtained the speed of light and mass.

\section{On the Zero Rest Mass and Vacuum-Pairs}

Here I will try to give their understanding of zero rest mass, as such, having in mind the rest mass of the photon and the rest mass of the leptons. Consider a pair of hypothetical particles with opposite signs unit charges. We assume that the particles are spherical radius $r_{e}$ and centers $n r_{e}$ spaced from each other (Figure 2). According to [11], the electrostatic energy of a sphere with a single charge

$$
E_{e l}=\frac{m_{e}}{2} c^{2}=\frac{1}{2} \cdot \frac{e^{2}}{4 \pi \varepsilon_{0} r_{e}},
$$

and the sum of their electrostatic energy twice as much

$$
2 E_{e l}=m_{e} c^{2}=\frac{e^{2}}{4 \pi \varepsilon_{0} r_{e}} .
$$

The internal energy of a charged sphere is the electrostatic energy mutual repulsion part of the charge. Recall that the repulsion energy of a positive and negative energy of attraction is. Real physical meaning of negative energy of attraction is manifested in the mass defect core elements. Consequently, when approaching oppositely charged particles, the energy of the Coulomb attraction will offset part of the internal energy and the mass of the system will decrease. 


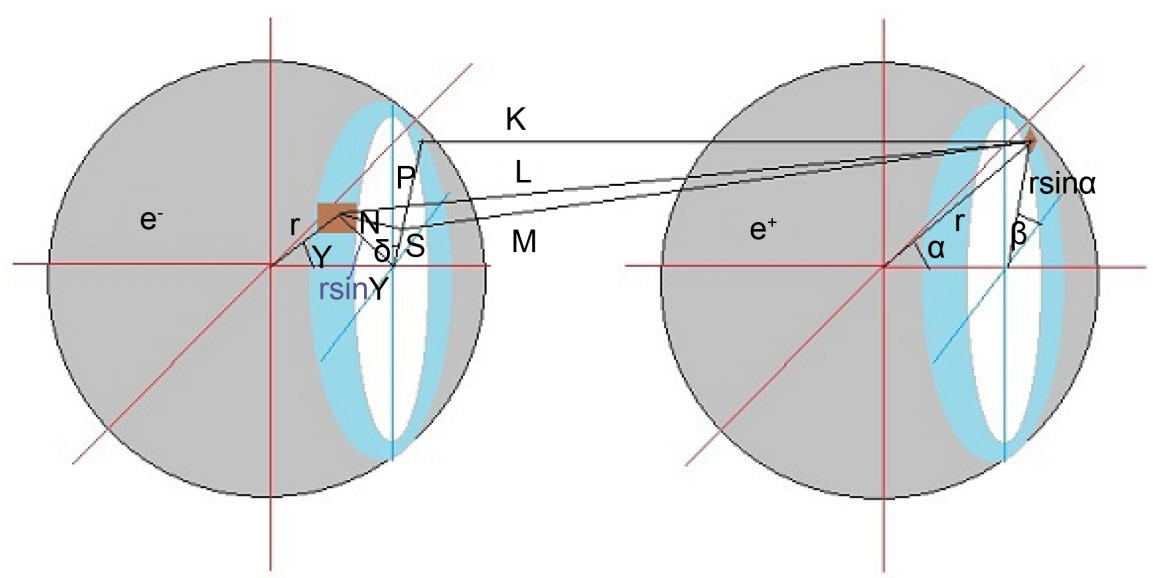

Figure 2. Scheme for calculating the interaction energy of a vacuum pair.

According to Coulomb's law for each of these particles is the force of attraction antiparticles

$$
F=\frac{e_{1} \cdot e_{2}}{4 \pi \varepsilon_{0}\left(2 r_{e}\right)^{2}}
$$

The energy of attraction equal to

$$
E=-F \cdot 2 r_{e}=-\frac{e^{2}}{8 \pi \varepsilon_{0} r_{e}}
$$

if the particles are in contact.

When the distance between the centers of gravity of less than $2 r$ energy should be calculated by integration.

We calculate the energy of attraction infinitesimal charges distributed on each of the sphere with surface density $\rho=\frac{e}{4 \pi r_{e}^{2}}$.

Figure 2 shows an arbitrary section of the right and left spheres. The angles between the line connecting the centers of the spheres and the radius vectors from the center of the right and left spheres are designated respectively $\alpha, \gamma$, rotation angles of the radii of the right and left sections $\beta, \delta$. Each of the sections are cut from a spherical surface strip width $r_{e} \mathrm{~d} \alpha, r_{e} \mathrm{~d} \gamma$, consisting of portions of width $r_{e} \sin (\alpha) \mathrm{d} \beta, r_{t} \sin (\gamma) \mathrm{d} \delta$. Land area, respectively $S_{1}=r_{e}^{2} \sin (\alpha) \mathrm{d} \beta \mathrm{d} \alpha, S_{2}=r_{e}^{2} \sin (\gamma) \mathrm{d} \delta \mathrm{d} \gamma$, the energy of attraction between them

$$
-\mathrm{d} E=-\frac{\rho^{2} \mathrm{~d} S_{1} \mathrm{~d} S_{2}}{4 \pi \varepsilon_{0} L}=\frac{e^{2} r_{e}^{4} \sin (\alpha) \sin (\gamma)}{64 \pi^{3} r_{e}^{4} \varepsilon_{0} L} \mathrm{~d} \delta \mathrm{d} \gamma \mathrm{d} \beta \mathrm{d} \alpha
$$

$$
\begin{gathered}
L=\sqrt{M^{2}+N^{2}}, N=r_{e e} \sin (\gamma) \sin (\delta), M=\sqrt{K^{2}+P^{2}}, K=r_{e}(n+\cos (\alpha)-\cos (\gamma)) \\
P=r_{e} \sin (\alpha)-S=r_{e} \sin (\alpha)-r_{e} \sin (\gamma) \cos (\delta)
\end{gathered}
$$

From Where

$$
L=\sqrt{r_{e}^{2}(n+\cos (\alpha)-\cos (\gamma))^{2}+r_{e}^{2}(\sin (\alpha)-\sin (\gamma) \cos (\delta))^{2}+r_{e}^{2}(\sin (\gamma) \sin (\delta))^{2}} .
$$

The energy of attraction spheres: 
$E_{n p}=-\frac{e^{2}}{64 \pi^{3} \varepsilon_{0} r_{e}} \int_{0}^{\pi} \int_{0}^{2 \pi} \int_{0}^{\pi} \int_{0}^{2 \pi} \frac{\sin (\beta) \sin (\phi)}{\sqrt{(n+\cos (\alpha)-\cos (\gamma))^{2}+((\sin (\alpha)-\sin (\gamma)) \cos (\delta))^{2}+(\sin (\gamma) \sin (\delta))^{2}}} \mathrm{~d} \delta \mathrm{d} \gamma \mathrm{d} \beta \mathrm{d} \alpha$

Calculations using this integral showed that full payment of the internal energy of the charged sphere with sufficient accuracy is achieved when the distance between the centers of the spheres $n=8.8995 \times 10^{-6} r_{e}$, and with the full combination negative energy of attraction slightly greater than the positive internal energy auto repulsion charges. Thus, due to the mutual electrostatic attraction of opposite elementary charges may form a system with zero energy and mass. We call such a hypothetical system of vacuum-pair because it is not a unitary entity capable divided into oppositely charged particles, and remains a combination of the two "retracted" into each other oppositely charged spheres. Physically, it is quite difficult to imagine, but remember that the electromagnetic radiation of any ranges, i.e., photons of different sizes are freely distributed in different directions without interfering with each other. The main properties of the vacuum-pairs are the absence of inertia and the ability to polarize.

Vacuum pairs can overcome the mutual attraction and disperse some distance, receiving positive energy from the outside. Photons are born, if the particles form a coherent system. The electron and positron are born with the full separation of vacuum pair Zero rest mass allows them to acquire the speed of light. Photons are able to spread in vacuo at a maximum speed along geodetic lines. Electron and positron is capable of orbital rotation of the speed of light, although the question of the radiation remains open.

\section{The Structure and Properties of the Electron}

Suppose that such a particle with a mass equal to half of the known mass of the electron, exist. We call it conditionally protoelektron. Assume also that the particle rotates at the speed of light in an orbit having the Compton radius. Moment of its rotation is equal to the characteristic fermion spin of the electron:

$$
s=\frac{1}{2} m_{e} R_{c e} c=5.27285863 \times 10^{-35} \mathrm{~J} \cdot \mathrm{s}=\frac{1}{2} \hbar .
$$

This gives us reason to believe the electron is not a unitary particle, but the system consisting of protoelektron that rotates at the speed of light in the orbit of the Compton radius. Rotational speed, or, more simply, the number of revolutions per second is equal to the quotient of the linear velocity by the circumference:

$$
v=\frac{c}{\lambda}=1.235589964 \times 10^{20} \mathrm{~s}^{-1} .
$$

The period of revolution $T$ is equal to:

$$
T=\frac{1}{v}=8.093299795 \times 10^{-21} \mathrm{~s} .
$$

With this understanding of the frequency of an electron becomes an identity known formula: 


$$
h v=m_{e} 2 \pi R_{c e} c \frac{c}{2 \pi R_{c e}}=m_{e} c^{2} .
$$

Electrostatic energy protoelektron as has been said above, is one-half of the total energy of the electron is determined by the formula

$$
E_{e l}=m_{p e} c^{2}=\frac{1}{2} m_{e} c^{2}=\frac{1}{2} \frac{e^{2}}{4 \pi \varepsilon_{0} r_{e}}=4.09355253 \times 10^{-14} \mathrm{~J}=0.255499464 \mathrm{MeV}=\frac{1}{2} E_{e} .
$$

It is the potential energy auto repulsion parts charge distributed on the sphere of classical electron radius. Mass of the protoelektrona

$$
m_{p e}=\frac{1}{2} \frac{e^{2}}{4 \pi \varepsilon_{0} r_{e} c^{2}}=4.554691455 \times 10^{-31} \mathrm{~kg}=\frac{1}{2} m_{e}
$$

Verify the possibility of get in this model, other well-known properties of the electron. The magnetic moment is by definition equal to the product of the current by the area covered the current circuit [12]. Rotating the charge at the speed of light in an orbit is equivalent to ring current:

$$
I_{e}=\frac{e c}{2 \pi R_{c e}}=19.7963328375 \mathrm{~A} .
$$

The magnetic moment of the electron $\mu$ :

$$
\mu=\frac{e c}{2 \pi R_{c e}} \pi R_{c e}^{2}=\frac{e c R_{c e}}{2}=9.27400968 \times 10^{-24} \mathrm{~J} \cdot \mathrm{T}^{-1} .
$$

The resulting magnetic moment is numerically equal to the Bohr magneton, having the known theoretical formula [5]. This formula (SI) can easily be obtained, considering that

$$
R_{c e}=\frac{h}{2 \pi \cdot m_{e} c} .
$$

Then

$$
\mu=\frac{e c}{2} \frac{h}{2 \pi \cdot m_{e} c}=\frac{e h}{4 \pi \cdot m_{e}}=\mu_{B}
$$

Anomalous magnetic moment is not considered here.

Try to find out the origin of the second half of the mass of the electron. Some part of the electron mass is due to the magnetic energy orbits charge equivalent to the ring current. Do the following thought experiment: imagine a stack of $10^{15}$ such rings, which is equivalent to the solenoid $N=10^{15}$ coils of the area $S=\pi R_{c e}^{2} m^{2}$, length $l=2 r_{e} \cdot 10^{15}=2 \times 2.8179403267 \mathrm{~m}$ with current 19.7963328375 A. Solenoid is long enough to hold the demagnetization coefficient equal to 1 . The inductance of the coil [5] $L$ is equal to:

$$
L=\frac{\mu_{0} N^{2} S}{l}=0.10445539459 \mathrm{H}
$$

The magnetic energy of the solenoid

$$
W_{m}=\frac{L I_{e}^{2}}{2}=20.467762664 \mathrm{~J} .
$$


The magnetic energy of one ring, i.e. electron:

$$
E_{m}=W_{m} \cdot 10^{-15}=20.467762664 \times 10^{-15} \mathrm{~J}=0.127749732 \mathrm{MeV}=\frac{1}{4} m_{e} c^{2}
$$

Here we have implicitly assumed accessory of all magnetic energy ring with current excluding magnetic scattering of energy in space related to the ratio between the height and the area of the ring. Thus, the formula of inductance and magnetic energy of the electron take the form

$$
\begin{gathered}
L_{e}=\frac{\mu_{0} \pi R_{c e}^{2}}{2 r_{e}}, \\
E_{m}=\frac{\mu_{0} \pi R_{c e}^{2}}{2 r_{e}} \frac{I_{e}^{2}}{2} .
\end{gathered}
$$

In this form, they will be used hereinafter.

Magnetic energy is considered the kinetic energy of motion of the charge. But there is the kinetic energy of rotation of the actual mass protoelektron. It accounts there are only $25 \%$ the total energy of the electron. Indeed, it turns out to be

$$
E_{k}=\frac{m_{p e} c^{2}}{2}=\frac{1}{4} m_{e} c^{2}
$$

The total energy of the electron is equal to the sum of Coulomb, the magnetic and kinetic energies:

$$
E_{e}=E_{e l}+E_{m}+E_{k}=\frac{1}{2} m_{e} c^{2}+2 \cdot \frac{1}{4} m_{e} c^{2}=m_{e} c^{2} .
$$

Accordingly, the Coulomb mass is half of the mass of the electron, and magnetic and kinetic masses are of $1 / 4$ the mass of the electron. The positron, which has a positive charge, is similar to the electron in all other respects.

\section{Checking}

This hypothesis, like any other, needs to be tested. The hypothesis is of particular value if it describes or explains a class of phenomena or objects and has a predictive capacity. In this case, we can say lucky-screening tests are not required. There are particles belonging to the same class of leptons as the electron (positron). It is muon and tau lepton with corresponding antiparticles. Their properties are studied. Therefore, we try to verify the applicability of the proposed model to them, and on its basis to calculate any properties in other known.

\subsection{Muon}

So, consider the muon. This is an unstable particle with a lifetime of about $2.2 \times 10^{-6} \mathrm{~s}$, decaying on an electron, an electron antineutrino and muon neutrino. Muon charge equal to the charge of the electron, spin is $\frac{1}{2} \hbar$, antimuon is antiparticle with positive charge, which decays into an positron, electron neutrino and muon antineutrinos. According to [4], rest mass of the muon 
$m_{\mu}=1.883531475 \times 10^{-28} \mathrm{~kg}$. The equivalent energy is equal

$E_{\mu}=1.69283367 \times 10^{-11} \mathrm{~J}=105.6583715 \mathrm{MeV}$. Compton wavelength of the muon $\lambda_{\mu}=11.734444103 \times 10^{-15} \mathrm{~m}$, the Compton radius

$R_{c \mu}=1.867594294 \times 10^{-15} \mathrm{~m}$. By analogy with the above models of electrons imagine a muon as protomuon with a mass equal to half the mass of the muon. Protomuon rotates at the speed of light in an orbit having a radius Compton.

According to this model muon spin really is

$$
s_{\mu}=\frac{1}{2} m_{\mu} R_{c \mu} c=5.27285863 \times 10^{-35} \mathrm{~J} \cdot \mathrm{s}=\frac{1}{2} \hbar .
$$

The expected radius protomuon

$$
r_{\mu}=\frac{1}{2} \frac{e^{2}}{4 \pi \varepsilon_{0} \frac{1}{2} m_{\mu} c^{2}}=1.362849403 \times 10^{-17} \mathrm{~m} .
$$

The ratio of the radius protomuon to Compton radius of the muon, i.e. the radius of its orbit, as expected, is equal to

$$
\frac{r_{\mu}}{R_{c \mu}}=7.29735257 \times 10^{-3}=\alpha .
$$

Expected muon magneton:

$$
\mu_{\mu}=\frac{e c R_{c \mu}}{2}=4.48521866 \times 10^{-26} \mathrm{~J} \cdot \mathrm{T}^{-1} .
$$

This value is within 8 decimal places coincide with the value calculated according to the accepted formula:

$$
\mu_{\mu}=\frac{e h}{4 \pi \cdot m_{\mu}} .
$$

Tabulated values of the anomalous magnetic moment of the muon $4.49044807 \times 10^{-26} \mathrm{~J} \cdot \mathrm{T}^{-1}$, anomaly is $1.16592091 \times 10^{-3}$. From way back it can also be obtained muon magneton. The calculated value is almost identical to the expected:

$$
\mu_{\mu}=\frac{4.49044807 \times 10^{-26}}{1+1.16592091 \times 10^{-3}}=4.4852186597 \times 10^{-26} \mathrm{~J} \cdot \mathrm{T}^{-1} .
$$

Equivalent ring current:

$$
I_{\mu}=\frac{c e}{2 \pi R_{c \mu}}=4.09325377689914 \times 10^{3} \mathrm{~A}
$$

Inductance:

$$
L_{\mu}=\frac{\mu_{0} \pi R_{\mu}^{2}}{2 r_{\mu}}=5.051809324316075 \times 10^{-19} \mathrm{H}
$$

The magnetic energy of the muon, indeed, is $1 / 4$ of its total energy:

$$
E_{m \mu}=\frac{\mu_{0} \pi R_{c \mu}^{2}}{2 r_{\mu}} \cdot \frac{I_{\mu}^{2}}{2}=4.232084168 \times 10^{-12} \mathrm{~J}=26.4145929 \mathrm{MeV}=\frac{1}{4} E_{\mu} .
$$

where the magnetic mass of the muon: 


$$
m_{m \mu}=\frac{E_{m \mu}}{c^{2}}=4.70882869 \times 10^{-29} \mathrm{~kg}=\frac{1}{4} m_{\mu} .
$$

The kinetic energy of the muon and the associated mass are also equal, respectively, $1 / 4$ of the total energy and the mass of the muon:

$$
\begin{gathered}
E_{k \mu}=\frac{1}{2} m_{\mu} \cdot \frac{c^{2}}{2}=4.232084168 \times 10^{-12} \mathrm{~J}=26.4145929 \mathrm{MeV}=\frac{1}{4} E_{\mu} \\
m_{k \mu}=\frac{E_{k \mu}}{c^{2}}=4.70882869 \times 10^{-29} \mathrm{~kg}=\frac{1}{4} m_{\mu}
\end{gathered}
$$

Thus, the structure of the muon fully explained by the hypothesis proposed for the electron.

\subsection{Tau Lepton}

The heaviest of the charged leptons, tau lepton [13] [14] has, according to the table http://physics.nist.gov/cuu/Constants/Table/allascii.txt rest mass $m_{\tau}=3.16747 \times 10^{-27} \mathrm{~kg}$, which corresponds to the equivalent energy $E_{\tau}=2.84678 \times 10^{-10} \mathrm{~J}=1776.82 \mathrm{MeV}$. Compton wavelength of the tau lepton $\lambda_{c \tau}=0.697787 \times 10^{-15} \mathrm{~m}$, the Compton radius $R_{c \tau}=0.111056 \times 10^{-15} \mathrm{~m}$. The lifetime of the tau lepton $290.6 \times 10^{-15} \mathrm{~s}$. It decays into an electron and an electron antineutrino with a tau neutrino (17.84\%), muon, muon antineutrino and a tau neutrino (17.36\%), as well as pi- and K-mesons (over 50\%). Charge tau lepton -1 , spin $1 / 2$. Assuming a structure the tau lepton similar to electron and muon, do the calculations similar to the above.

$$
\begin{gathered}
S_{\tau}=\frac{1}{2} m_{\tau} R_{\tau} c=5.2728 \times 10^{-35} \mathrm{~J} \cdot \mathrm{s}=\frac{1}{2} \hbar \\
r_{\tau}=\frac{1}{2} \frac{e^{2}}{4 \pi \varepsilon_{0} \frac{1}{2} m_{\tau} c^{2}}=8.1041 \times 10^{-19} \mathrm{~m} \\
\frac{r_{\tau}}{R_{c \tau}}=7.2973 \times 10^{-3}=\alpha \\
\mu_{\tau}=\frac{e c R_{c \tau}}{2}=2.66712 \times 10^{-27} \mathrm{~J} \cdot \mathrm{T}^{-1}=\frac{e \hbar}{2 m_{\tau}} \\
L_{\tau}=\frac{\mu_{0} \pi R_{\tau}^{2}}{2 r_{\tau}}=3.0041 \times 10^{-20} \mathrm{H} \\
I_{\tau}=\frac{e c}{2 \pi R_{c \tau}}=6.8834 \times 10^{4} \mathrm{~A} \\
E_{m \tau}=\frac{\mu_{0} \pi R_{c \tau}^{2}}{2 r_{\tau}} \cdot \frac{I_{\tau}^{2}}{2}=7.116 \times 10^{-11} \mathrm{~J}=\frac{1}{4} E_{\tau} \\
E_{k \tau}=\frac{1}{2} m_{\tau} \cdot \frac{c^{2}}{2}=\frac{m_{\tau} c^{2}}{4}=\frac{1}{4} E_{\tau}
\end{gathered}
$$

As you can see, the tau lepton is completely analogous to the structure and properties of the electron and muon. 


\section{On Heisenberg's Uncertainty Principle}

Werner Heisenberg Uncertainty Principle is, along with the Schrödinger wave equation, one of the foundations of quantum mechanics. It is called the principle because, just as the law describes the establishment of relations, but, unlike the law, the physical nature of these relations remains undisclosed. Here I will try at least to slightly open the physical meaning of the principle on the basis of the above suggested model of the electron. The Heisenberg uncertainty principle is written as:

$$
\Delta P \cdot \Delta X \geq \frac{\hbar}{2}
$$

i.e. the product of the position and momentum of a particle cannot be less than half of the reduced Planck constant. Consider an electron at rest as a boundary case. "Resting" electron in this hypothesis should be understood both at rest the center of the orbit on which revolves protoelektron relative to the observer. If you take as the origin center of the orbit, the coordinates of protoelektron be considered the radius of the orbit $R_{c e}$. Linear speed protoelektrona as particles with zero rest mass is equal to the speed of light, the mass is half the mass of the electron. Momentum is protoelektrona

$$
P_{p e}=\frac{m_{e}}{2} \cdot c .
$$

The limit case is the union of the formulas (5.1) and (1.19):

$$
P_{\min } \cdot X_{\min }=\frac{m_{e}}{2} \cdot R_{c e} \cdot c=\frac{\hbar}{2} .
$$

Thus, by virtue of the uncertainty principle rotating at the speed of light protoelektron cannot be on a distance less than the Compton radius from the center of the orbit. As you can see, on the one hand the structure of the electron explains the origin of the uncertainty principle and the spin, the other spin and the uncertainty principle completely describe the structure of the electron. Similar expressions exist for the muon and tau lepton:

$$
\begin{gathered}
P_{\min } \cdot X_{\min }=\frac{m_{\mu}}{2} \cdot R_{c \mu} \cdot c=\frac{\hbar}{2} . \\
P_{\min } \cdot X_{\min }=\frac{m_{\tau}}{2} \cdot R_{c \tau} \cdot c=\frac{\hbar}{2} .
\end{gathered}
$$

\section{The Wave Properties of a Moving Electron}

Since in this model electron "at rest" corresponds to the orbital rotation protoelektron, thus in forward motion of an electron protoelektron will perform more complex periodic motion. The translational motion of an electron is caused, as a rule, by an external electric field. In this regard, it can be excluded from consideration rotation about an axis normal to the direction of movement, since the trajectory protoelektron then will have the form of a cycloid, comprising plots directed against the external fields. Thus, during the forward movement of the orbit center protoelektron moves in a spiral-screw line, the helicity of which 
coincides with the direction of movement (Figure 3).

Thus, naturally, a problem arises: how to combine orbital rotation, has the speed of light, with translational motion having any speed not exceeding the speed of light? The only way to solve this problem appears to decrease radius of rotation with increasing of forward speed. Changing this geometric parameters due to physical causes, and especially the growth of the total energy and the total mass of the electron due to forward speed. In modern physics [15], all less used notion of relativistic mass and becoming more popular concept of the invariant mass of rest at the expense of variability abstract time. However, we have seen that the mass is equivalent to the algebraic sum of the energies. The behavior of a moving electron again testifies to the primacy of the properties of matter.

Protoelektron acquiring translational movement, cannot increase their speed, so increasing its mass, resulting in the multiplication of the rest mass protoelektrona on the Lorentz:

$$
m_{p e}(\vartheta)=m_{p e} \cdot \frac{1}{\sqrt{1-\frac{\vartheta^{2}}{c^{2}}}}=\frac{m_{e}}{2} \cdot \frac{1}{\sqrt{1-\frac{\vartheta^{2}}{c^{2}}}} .
$$

Here $\vartheta$-the speed of translational motion of an electron as a whole. From Figure 3 that the radical expression is not hyperbolic sine in a pseudo-Minkowski space and the trigonometric sine of the angle $\theta$ in real Euclidean space.

$$
\theta=\arccos \frac{\vartheta}{c} \text {. }
$$

Accordingly, own protoelektron radius decreases:

$$
\frac{e^{2} \sqrt{1-\frac{\vartheta^{2}}{c^{2}}}}{4 \pi \varepsilon_{0} m_{e} c^{2}}=r_{e} \sqrt{1-\frac{\vartheta^{2}}{c^{2}}} .
$$

But, as we already know, own radiuses charged protoleptons rigidly connected with the radii of their rotation through the fine-structure constant. Consequently, as a result of the growth of the relativistic energy and mass protoelektron as

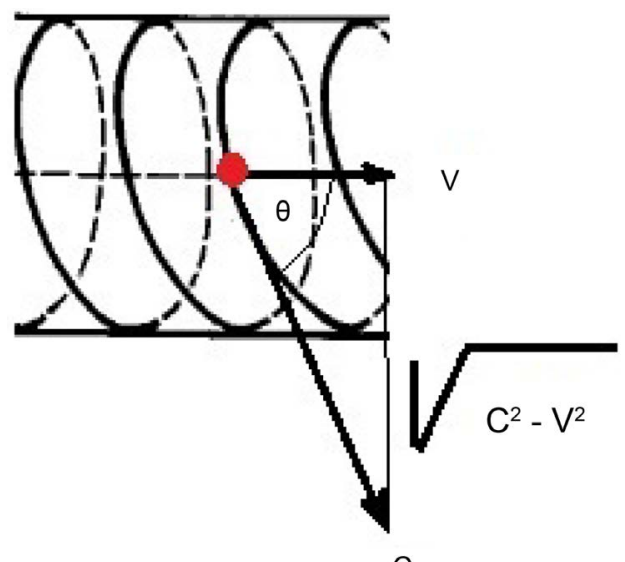

C

Figure 3. Ratio of rotational speed and translational motion of a potoelectron. 
many times decreases the radius of rotation. Figure 4 illustrates a method of calculating the length of the screw line [16].

Side $B C$ angled triangle is equal to the circumference of the cylinder, in this case,

$$
B C=2 \pi R_{c e} \sqrt{1-\frac{\vartheta^{2}}{c^{2}}}=\lambda_{c e} \sqrt{1-\frac{\vartheta^{2}}{c^{2}}}=\lambda_{c e} \sin \theta,
$$

where $R_{c e}$-the radius of Compton, $\lambda_{c e}$-Compton wavelength of the electron. If a paper triangle $A B C$ is wound on the cylinder then side $A B$ forms a helix. However, since the rate of protoelektron remains equal speed of light, the path length for one revolution at any forward speed remains equal to the Compton wavelength. According to the theorem of Pythagoras,

$$
A B=\lambda_{c e}=\sqrt{A C^{2}+B C^{2}} .
$$

Consequently, in view of (6.4), a step helix

$$
A C=\lambda_{c e} \cos \theta .
$$

The frequency of the electron in the electric field is constant and independent of forward speed:

$$
v=\frac{\vartheta}{A C}=\frac{C}{\lambda_{c e}} .
$$

Forward speed only leads to a reduction in the rotation radius protoelektrona and to increase the step helix. The trajectory translational motion of protoelektron looks stretched spring whose modulus of deformation under tension tends to infinity. Moving electron physically embodies Einstein invariant interval: real part of the complex number describes the pathway the center of the orbit, and the imaginary part, the length of the helical path of protoelektron.

Consider what is the de Broglie wavelength [17]. The relativistic momentum formula is:

$$
p=\frac{m \vartheta}{\sqrt{1-\frac{\vartheta^{2}}{c^{2}}}} .
$$

With this in mind, the de Broglie wavelength is of an electron can be expressed through the Compton wavelength:

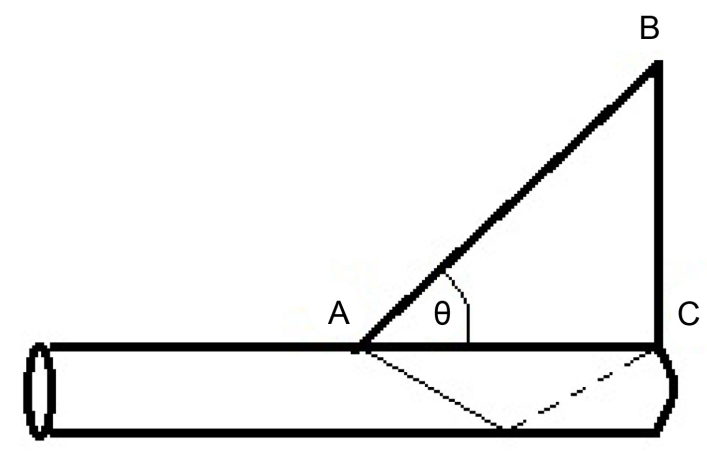

Figure 4. To calculate the wavelength of a divalent electron. 


$$
\lambda_{B}=\frac{h}{p}=\frac{m_{e} \lambda_{c e} c}{m_{e} \vartheta} \sqrt{1-\frac{\vartheta^{2}}{c^{2}}}=\lambda_{c e} \operatorname{tg} \theta
$$

Note that a decrease of the electron rate de Broglie wavelength approaches infinity, while increasing to zero, which corresponds to photons emitted when stopping electrons in the target. Check this prediction using a magnet [17] must be repeated with approaching of the magnet no to the target and the screen.

\section{The Mechanism of Rotation of Leptons}

Naturally, the question arises, what is the reason for the rotation of protoelektron in the spin orbit? The first candidate is the intrinsic magnetic field. From the equality of the classical centripetal force $F_{\mathrm{L}}=\frac{m v^{2}}{r}$ and the Lorentz force [18] $\quad F_{л}=q[B \cdot v]$ should be known [19] formula relating the magnetic induction $\mathrm{B}$ with the radius of rotation $\mathrm{r}$ of the charged particle in a uniform magnetic field: $B=\frac{m v}{q r}$. When $m=\frac{m_{e}}{2}, q=e, \quad v=c, \quad r=R_{c e}$

$$
B_{1}=\frac{m_{e} C}{2 e R_{c e}}=2.207002512 \times 10^{9} \mathrm{~T}
$$

Using the values of inductance (40) and current (33), we find the magnetic flux through the area bounded of the orbit of the spin:

$$
\Phi=L \cdot I=\frac{\mu_{0} \pi R_{c e}^{2}}{2 r_{e}} \cdot \frac{e \cdot c}{2 \pi R_{c e}}=\frac{\mu_{0} R_{c e} e c}{4 r_{e}}=2.0678337580690105 \times 10^{-15} \mathrm{~W}
$$

The flow through the orbit of the spin turned out to be well-known magnetic flux quantum [20]:

$$
\Phi_{0}=\frac{h}{2 e}=2.067833758 \times 10^{-15} \mathrm{~W}
$$

Formula of magnetic flux quantum can be expressed in other ways:

$$
\Phi_{0}=\frac{m_{e} \lambda_{c e} c}{2 e}=\frac{\pi \cdot c \cdot e \cdot 10^{-7}}{\alpha}
$$

The magnetic flux equal to the product of the magnetic induction in the area, from whence the magnetic induction

$$
B_{2}=\frac{\Phi_{0}}{\pi R_{c e}^{2}}=4.414005024 \times 10^{9} \mathrm{~T}=2 B_{1}
$$

Magnetic induction twice as much required to rotate protoelektron. This suggests that the kinetic and magnetic energy is localized on protoelektron, increasing its mass. This is confirmed, so how the magnetic induction and the Bohr magneton product is equal half the energy of the electron:

$$
B_{2} \cdot \mu_{\mathrm{b}}=4.093552533 \times 10^{-14} \mathrm{~J}=E_{M}+E_{\kappa} .
$$

Whence appears such an induction? It turns the same value may be obtained from the formulae 


$$
B_{3}=\frac{c \cdot e}{2 \pi r_{e}} \cdot \frac{\mu_{0}}{2 R_{c e}}=4.414005025 \times 10^{9} \mathrm{~T}=B_{2}
$$

As such the physical meaning of this formula is unclear. However note that fractional expression on the left-a current produced by the rotation of the elementary charge the speed of light in the orbit of classical radius, the current of giant force localized in vanishingly small space:

$$
I_{1}=\frac{c \cdot e}{2 \pi r_{e}}=2.712810248 \times 10^{3} \mathrm{~A}
$$

This current can be interpreted in different ways, such as rotation of a point charge or charged ring. In this hypothesis is regarded a sphere of classical radius. We check whether you can get such current by rotation of the charged sphere. Assume that the speed of light rotate only points on the equator of the sphere. Then the angular frequency is $\varpi=\frac{c}{2 \pi r_{e}}$. The surface charge density is

$$
\begin{gathered}
\rho=\frac{e}{4 \pi r_{e}^{2}} . \quad \mathrm{d} I_{2}=\varpi \rho \cdot 2 \pi r_{e} \sin \varphi \cdot r_{e} \mathrm{~d} \varphi=\frac{c \cdot e}{4 \pi r_{e}} \sin \varphi \mathrm{d} \varphi . \\
I_{2}=\frac{c \cdot e}{4 \pi r_{e}} \int_{0}^{\pi} \sin \varphi \mathrm{d} \varphi=2.712810248 \times 10^{3} \mathrm{~A}=I_{1}
\end{gathered}
$$

The final

$$
B_{4}=I_{2} \cdot \frac{\mu_{0}}{2 R_{c e}}=4.414005024 \times 10^{9} \mathrm{~T}=B_{2} .
$$

This equation shows that the magnetic induction in the center of the orbit of spin equal the induction of a uniform magnetic field outside the sphere protoelektron. For the appearance of the required Lorentz force should be rotation axis of protoelektron parallel to of spin and the direction of own rotation must be opposite to spin.

What is the cause protoelektrona rotation around its own axis? For a own rotation of the charge requires a uniform magnetic field of induction

$$
B_{5}=\frac{m_{e}}{e} \cdot \frac{c}{r_{e}}=6.048775884 \times 10^{11} \mathrm{~T}
$$

It turns out that current creates on the axis of a sphere induction just such:

$$
B_{6}=\frac{I_{2} \cdot \mu_{0}}{2 r_{e}}=6.048775884 \times 10^{11} \mathrm{~T}=B_{5} .
$$

Thus, we have a fully self-acting system: protoelektrona own rotation is due to his own field, and provides orbital rotation. This passes through the body protoelektron magnetic flux in 137.036 times less is known quantum flux:

$$
\Phi_{01}=B_{5} \cdot \pi r_{e}^{2}=1.508971198 \times 10^{-17} \mathrm{Vb}=\Phi_{0} \alpha .
$$

At the same time the magnetic induction on the axis protoelektrona to 137.036 times higher induction in the center of the orbit of spin.

$$
\frac{B_{5}}{B_{2}}=137.03599907 \cong \frac{1}{\alpha}
$$


This result explains the origin of the fine structure constant and its geometrical expression-the relation between classical and Compton radius.

Here we are faced with a new phenomenon microcosm: own rotation protoelektrona provides rotation of the orbit of spin, so there are magnetic and kinetic electron mass, spin and magnetic moment. However, it makes no contribution to these values.

For short-lived charged leptons regularities is characterized by, similar to the above for elektron. For the muon using (4.1.7) and (4.1.8), we obtain

$$
\begin{gathered}
L_{\mu} \cdot I_{\mu}=2.067833759 \times 10^{-15} \mathrm{~W} \cong \Phi_{0} \\
B_{2}^{\mu}=\frac{\Phi_{0}}{\pi R_{c \mu}^{2}}=\frac{m_{\mu} c}{e R_{c \mu}}=1.88712501 \times 10^{14} \mathrm{~T} \\
I_{2}^{\mu}=\frac{c \cdot e}{4 \pi r_{\mu}} \int_{0}^{\pi} \sin (\varphi) \mathrm{d} \varphi=5.60923121 \times 10^{5} \mathrm{~A} \\
B_{4}^{\mu}=I_{2}^{\mu} \frac{\mu_{0}}{2 R_{c \mu}}=1.88712501 \times 10^{14} \mathrm{~T} \\
B_{5}^{\mu}=B_{6}^{\mu}=\frac{m_{\mu} c}{e r_{\mu}}=I_{2}^{\mu} \frac{\mu_{0}}{2 r_{\mu}}=2.58604062 \times 10^{16} \mathrm{~T} \\
\frac{B_{5}^{\mu}}{B_{2}^{\mu}}=137.036999 \cong \frac{1}{\alpha}
\end{gathered}
$$

Accordingly, for the tau lepton, using (4.2.5) and (4.2.6), we find

$$
\begin{gathered}
L_{\tau} \cdot I_{v}=2.0678 \times 10^{-15} \mathrm{~W} \cong \Phi_{0} \\
B_{2}^{\tau}=\frac{\Phi_{0}}{\pi R_{c \tau}^{2}}=\frac{m_{\tau} c}{e R_{c \tau}}=5.3368 \times 10^{16} \mathrm{~T} \\
I_{2}^{\tau}=\frac{c \cdot e}{4 \pi r_{v}} \int_{0}^{\pi} \sin (\varphi) \mathrm{d} \varphi=9.4329 \times 10^{5} \mathrm{~A} \\
B_{4}^{\tau}=I_{2}^{\tau} \frac{\mu_{0}}{2 R_{c \tau}}=5.3368 \times 10^{16} \mathrm{~T} \\
B_{5}^{\tau}=B_{6}^{\tau}=\frac{m_{\tau} c}{e r_{\tau}}=I_{2}^{\tau} \frac{\mu_{0}}{2 r_{\tau}}=7.3134 \times 10^{18} \mathrm{~T} \\
\frac{B_{5}^{\tau}}{B_{2}^{\tau}}=137.037 \cong \frac{1}{\alpha}
\end{gathered}
$$

It is known that the movement of electrons in a magnetic field takes place along a helix whose step of the screw

$$
h=\frac{2 \pi m \vartheta}{q B} \cos \alpha
$$

Under Section 6, the electron in the absence of a magnetic field is also moving along a helical path through the spin. Pitch is determined from (6.6). Hence, the same step of the screw must be obtained from (7.27) due to the precession angle $\theta$ of the intrinsic magnetic field of the electron.

$$
h_{e}=\frac{2 \pi m_{e} c}{e B_{2}} \cos \theta=2.426310239 \times 10^{-12} \cos \theta \cong \lambda_{c e} \cos \theta,
$$


this corresponds to (74).

\section{Acknowledgements}

The author is grateful to VA Vostrotyukov, VV Erokhin, AY Sergeeva, Doctor of Physics and Mathematics BA Kulik, the candidate of physical and mathematical sciences SA Startsev, AV Skripkin, VF Borulko, SF Lyagushin, SA Anfinogentov for your attention and help in the work, valuable comments and advice.

\section{References}

[1] Schunck, E. (2013) Magnetic Dipole Moment of the Electron. http://arxiv.org/pdf/0809.2770v1.pdf

[2] Trunev, A.P. (2010) Electron Structure in Classical and Quantum Electrodynamics. Chaos and Correlation, 7. http://chaosandcorrelation.org/Chaos/CR7_1_2010.pdf

[3] Abraham, M. (1903) Prinzipien der Dynamik des Elektrons. Annalen der Physik, 315, 105-179. https://de.wikisource.org/wiki/

[4] http://physics.nist.gov/cuu/Constants/Table/allascii.txt

[5] Karyakin, N.I., Bystrov, K.N. and Kireev, P.S. (1969) Quick Reference Book on Physics. 3rd Edition, High School, Moscow.

[6] Shulman, M.E. Structure of the Electron. http://new-idea.kulichki.net/pubfiles/090627010440.pdf

[7] Kiriako, A.G. (2006) The Theory of Nonlinear Waves, Adequate to Quantum Field Theory. http://www.partphys.envy.nu/Russian/Oglavlenie_knigi.htm

[8] Kholodov, I. and Goryachev, I.V. The Ideas of the Physical Meaning of the Fine Structure Constant. http://www.trinitas.ru/rus/doc/0016/001e/00162918.htm

[9] Nikolaev, G.V. (2003) Modern Electrodynamics and the Causes of Its Paradox. Prospects for Building Consistent Electrodynamics. Theories, Experiments, Paradoxes. 2nd Edition, Publishing House n.-t. Lit-ry, Tomsk.

[10] Landau, L.D. and Lifshitz, E.M. (1967) Field Theory. 5th Edition, Corrected and Additional, Science, Moscow.

[11] Feynman, R., Leighton, R. and Sands, M. Feynman Lectures on Physics. T.6. Electrodynamics.

[12] Physical Encyclopedia. Magnetic Moment. http://femto.com.ua/articles/part_1/2091.html

[13] Pearl, M. (1979) The Discovery of a New Elementary Particle-A Heavy Tau Lepton. UFN, 129.

[14] Azimov, Y.I. (1980) The Modern Status of Tau Lepton. UFN, 132.

[15] Ugarov, V.A. (1969) Special Theory of Relativity. Science, Moscow.

[16] Vinogradov, I.M. (1999) Elements of Higher Mathematics. (Analytical Geometry, Differential Calculus, Fundamentals of Number Theory). Textbook for Universities.

[17] Thomson, J.J. Outside the Electron.

[18] Physical Encyclopedia. The Power of Lorentz. http://femto.com.ua/articles/part_1/1986.html

[19] Yavorsky, B.M. and Detlaff, A.A. (1965) Handbook of Physics. For Engineers and University Students, 3rd Edition, Nauka, Moscow.

[20] Tomilin, K.A. To the History of the Discovery of the Quantization of Magnetic Flux. Conference Reports. 
Submit or recommend next manuscript to SCIRP and we will provide best service for you:

Accepting pre-submission inquiries through Email, Facebook, LinkedIn, Twitter, etc. A wide selection of journals (inclusive of 9 subjects, more than 200 journals)

Providing 24-hour high-quality service

User-friendly online submission system

Fair and swift peer-review system

Efficient typesetting and proofreading procedure

Display of the result of downloads and visits, as well as the number of cited articles Maximum dissemination of your research work

Submit your manuscript at: http://papersubmission.scirp.org/

Or contact jhepgc@scirp.org 